\title{
OPINION
}

\section{Does true Gleason pattern 3 merit its cancer descriptor?}

Saiful Miah ${ }^{1}$, Hashim U. Ahmed ${ }^{2}$, Alex Freeman ${ }^{2}$ and Mark Emberton ${ }^{1}$

${ }^{1}$ Division of Surgery and Interventional Science, University College London Medical School Building, 21 University Street, London WC1E 6AU, U.K. ${ }^{2}$ Department of Pathology, University College London Hospital, $3^{\text {rd }}$ Floor East, 250 Euston Road, London NW1 2PG, U.K.

Correspondence to M.E. m.emberton@ucl.ac.uk

\section{Competing interests statement}

The authors declare no competing interest.

\section{Author contributions}

All authors contributed to researching data for the article, discussing the content and writing the article. All authors were involved in reviewing and editing the manuscript before submission.

\begin{abstract}
Nearly five decades following its conception, the Gleason grading system remains a cornerstone in the prognostication and management of patients with prostate cancer. In the past few years, a debate has been growing whether Gleason score $3+3=6$ prostate cancer is a clinically significant disease. Clinical, molecular and genetic research is addressing the question whether well characterized Gleason score $3+3=6$ disease has the ability to affect the morbidity and quality of life of an individual in whom it is diagnosed. The consequences of treatment of Gleason score 3+3=6 disease are considerable; few men get through their treatments without sustaining some harm. We propose a further modification of the classification of prostate cancer and drop the label cancer for Gleason score $3+3=6$ disease.
\end{abstract}

\section{Introduction}

The American Cancer Society defines cancer as a disease that is caused by the abnormal and uncontrolled growth of cells, possesses the ability to invade other tissues and, if left untreated, will lead to serious morbidity and eventually death. The subject of this Perspective article is important, given that prostate cancer is 
the second most prevalent cancer in men ${ }^{1}$. Furthermore, as the overall male population ages the prevalence will increase. The International Agency for Research on Cancer reported that prostate cancer accounted for $14 \%$ of all cancers diagnosed in men and $>900,000$ men were diagnosed with the disease throughout the world in 2008 alone. Most of these cancers will have a $3+3=6$ Gleason pattern on diagnosis ${ }^{2}$.

Nearly five decades after its original conception, the Gleason grading system remains the cornerstone of the management of patients with prostate cancer ${ }^{3}$. To this day, this histological classification has upheld its position as the principal determinant of disease progression. This prognostic attribute is achieved in a rather unusual way. Most histological grading follows an ordinal scale based on the degree of cytological atypia within the cells of a tumour. The Gleason grading system does not incorporate any information on cytological parameters. Instead, it is principally established on the architectural pattern, size and spacing of the tumour glands rather than the nuclear features of the malignant cells themselves. In addition, the system's prognostic value has withstood despite changes to the way in which grade is determined and score calculated ${ }^{4}$.

Over the years, it has become widely accepted that Gleason patterns 1 and 2 diagnosed on prostatic needle biopsy specimens actually represent benign proliferations, such as adenosis or atypical adenomatous hyperplasia. A number of concerns mean that it is now time to evaluate whether Gleason pattern 3 that has been well characterised by modern techniques should be considered similar to pattern 1 and 2. Clearly, a change in designation of Gleason pattern 3 to a noncancer status would have profound consequences. In this Perspective article, we argue that most of these consequences are likely to be beneficial to those men diagnosed with Gleason score $3+3=6$ prostate cancer and the viewpoint of Gleason pattern 3 prostate cancer as a lethal disease is becoming increasingly uncertain $^{5-8}$.

\section{Evolution of Gleason grading}




\section{The birth of Gleason grading}

In 1966, Donald Gleason created a unique grading system for prostate cancer that was entirely derived from architectural features of the disease using lowpowered microscopy 3 With a five-point scale, patterns 1, 2 and 3 represented tumours that most closely resembled normal prostatic glands and patterns 4 and 5 demonstrated an increasingly abnormal glandular architecture. A derivative of the patterns was constructed from the sum of the two most commonly observed grade patterns and became known as the Gleason score. The original description of this system was based on a study in 270 patients, which a later study in 1974 expanded to include 1,032 men ${ }^{9}$. In his early studies, Donald Gleason reported that he could duplicate his grading scores during reexamination of specimens within \pm 1 score point in $85 \%$ of cases 3,10 . Furthermore, he reported that his grading score had a strong prognostic value and could predict cancer-specific mortality.

\section{Changes in scoring over time}

Many aspects of the clinical management of men with prostate cancer have changed since the conception of the Gleason grading system. To accommodate this change in clinical practice, substantial modifications of this system have occurred $^{11}$. For instance, Gleason pattern 1 and 2 are now considered to represent benign proliferations in most instances.

The problem with the low grades 1-3 that led to this re-evaluation concerned their reproducibility. Biopsy samples were based on a transrectal systematic approach that often did not characterize the cancer properly. Assessment of low-grade tumours had the greatest interobserver variability among urological pathologists $^{12,13}$.

In the contemporary assessment of prostatic needle biopsy samples, Gleason grading starts at Gleason pattern 3. Owing to the advent and regular use of immunohistochemistry, many of the specimens originally classified as Gleason pattern 1 would now be regarded as atypical adenomatous hyperplasia14,15. By the turn of the millennia, a proposal was made that Gleason patterns 1 and 2 should not be diagnosed as prostate cancer, regardless of the type of specimen, owing to inaccuracies and poor reproducibility between experts ${ }^{14,16}$. In 2001 , the 
rate of biopsy reports describing Gleason scores of 2-4 was $2.4 \%$ and reduced to $0 \%$ by $2006^{17,18}$.

In 2005, an adaptation of the Gleason grading system was accepted at a consensus conference of international experts in urological pathology under the auspices of the International Society of Urological Pathology (ISUP) ${ }^{15,19}$. This adjustment was made to address areas of debate and the existence of architectural arrangements that the original scoring system had not accounted for, aiming to increase reproducibility and reliability of the evaluation of biopsy specimens. The major changes were the inclusion of poorly formed glands into Gleason pattern 4 which would have been previously labelled as Gleason grade $\leq 3$. Furthermore the revised definition of Gleason score based on needle biopsy samples, which should always include the highest Gleason pattern detected in a specimen $^{19}$. In addition, the new agreement reclassified irregular cribiform glands from a pattern 3 to 4 . These modifications resulted in a statistical artefact termed the Will Rogers Phenomenon, with improvement of clinical outcomes for both Gleason Score 3+3=6 and 3+4=7 prostate cancer ${ }^{20,21}$. This clarification on which architectural elements should be attributed a pattern 4 label effectively removed disease with a poor clinical outcome from Gleason score $3+3=6$. In addition, the amendment resulted in an overall reduction in the risk of morbidity and mortality associated with attribution of Gleason pattern 4 compared with the classification used before 2005. Thus, any prediction models based on samples that were histologically graded before 2005 (which includes nearly all models) overestimate the risk associated with Gleason pattern 4 disease that is graded using the post-2005 rules.

The current definition of Gleason pattern 3 consists of disease with discrete glandular units that vary in size and shape within the prostatic stroma ${ }^{19}$. Furthermore, individual tumour acini demonstrate a smooth and usually rounded edge with an intact basement membrane. Gleason pattern 4 describes a disruption to the form associated with pattern 3: the glandular architecture is no longer preserved and the basement membrane is disrupted. In this sense, the threshold between Gleason pattern 3 and pattern 4 is not dissimilar from pTa and pT1 in bladder cancer. 


\section{Changes in biopsy techniques}

Over the past century, we have witnessed continued modifications to the process of tissue procurement for analysis by the pathologist. The methods have comprised open biopsy, endoscopic punch biopsy, transperineal (blind or ultrasound-guided) or transrectal (finger-guided or ultrasound-guided (TRUS)) biopsy, endoscopic resection and, most recently, image-guided biopsy with or without image registration ${ }^{22}$. At any time point, several different approaches have been in use. Current procedures include 12-core TRUS biopsy, transperineal template biopsy and biopsy targeted to an imaging-identified lesion $23-25$.

Techniques of prostate TRUS biopsy have progressed from systematic sextant to 12-core biopsies, which demonstrated increased cancer detection rates without increasing morbidity 22 . Increasing the number of biopsy cores increased prostate cancer detection rates including detection of disease without lethal potential $^{26}$. The original error was a random biopsy rather than basing the biopsy deployment on the phenotype presenting itself. Image-guided biopsy is used in the diagnosis of the majority of all cancers The visual area of concern is identified before sampling a specific area of abnormality.

When the Gleason grading system was first described the vast majority of men presented with advanced disease and tissue was obtained using a few low-gauge needles directed into the area of palpable abnormality. Now, most biopsies are performed using thin needles, resulting in specimens that differ from those available when the system was created. The use of thinner needles have been shown to decrease post-biopsy infection rates at the expense of providing less tissue per core. ${ }^{27}$

The biopsy method employed influences whether any Gleason pattern 4, if present, will be detected. A biopsy using a 10-12-core transrectal technique can miss pattern 4 in up to $50 \%$ of patients ${ }^{28}$. By contrast, the use of modern MRIguided techniques results in very few Gleason pattern 4 cancers being missed ${ }^{29}$. The consequence is that upgrading (the finding that Gleason score at definitive pathological assessment following radical prostatectomy is higher than at initial biopsy) is significantly reduced ${ }^{30}$. When standard TRUS biopsy is used during the 
diagnostic work-up, upgrading occurs in up to $50 \%$ of patients ${ }^{28}$, which means that risk stratification at diagnosis was incorrect in half of the patients.

\section{Upgrading}

\section{Sampling imprecision and reproducibility}

The driving force towards increased sampling density or image-guided biopsy is a result of the desire to reduce diagnostic imprecision. This problem most commonly becomes evident when patients are upgraded or reclassified when the pathological status of their radical prostatectomy specimen is compared to the risk attributed to their disease by the pathological assessment of their biopsy sample $31,32,33$. This phenomenon has been found in all investigations of this topic published to date, which report reclassification rates that range between $25 \%$ and 50\% ${ }^{34-37}$. Reclassification error has been shown to be related to the different manner in which Gleason patterns were declared upon assessment of biopsy specimen compared with the full histopathology available at step-sectioning following radical prostatectomy ${ }^{33}$. Gleason score upgrading, as well as downgrading, is still an important problem when using the latest Gleason system, even when accounting for tertiary Gleason patterns in radical prostatectomy specimens ${ }^{33}$.

Upgrading of disease merely represents sampling imprecision as opposed to a mandate for justifying the potential presence of increased-grade disease coexisting in the prostate. The occurrence of upgrading highlights a need for development in the manner of how we obtain tumour specimens and adoption of targeted biopsies using MRI or MRI-ultrasound fusion, which is expected to be declared the gold-standard procedure ${ }^{38}$. We now have level $1 \mathrm{~b}$ evidence to support the adoption of mpMRI prior to a first prostate biopsy as the standard of care $^{39}$ : In the presence of Gleason score $3+3=6$ prostate cancer, upgrading should not be confused as a rationale for treating patients with nonhistologically confirmed significant disease.

In addition, problems regarding the reproducibility of Gleason grading, even amongst pathologists, have been highlighted ${ }^{40}$, This inconsistency would be a potential source for inconsistent diagnoses of cancer in patients who are found 
to have prostatic tissue with a Gleason score $3+3=6$. An agreement on even stricter criteria separating Gleason pattern 3 from pattern 4 might be required before we are to consider any changes to the nomenclature of prostate cancer, and could also include a requirement for centralized review of all equivocal specimens.

\section{Capacity of Gleason pattern 3 to progress}

Evidence is accumulating that shows the lacking ability of low-grade prostate cancers to progress to higher-grade disease ${ }^{41}{ }^{42}$. One study in 1, 207 patients (including 267 men with Gleason score $\leq 6$ ) who underwent prostatectomy between 1982 and 2004 has reported data suggesting that grade is established early in tumour pathogenesis ${ }^{41}$.

Previous reports of Gleason pattern 3 progressing to higher grades have now been primarily attributed to the inherent sampling insufficiencies of using conventional TRUS-guided biopsy. In 2014, one team of investigators demonstrated that the progression of Gleason pattern 3 to a higher pattern was a rare occurrence in men on active surveillance when both initial and follow-up targeted biopsies were undertaken in an accurate manner using MRI-ultrasound fusion techniques ${ }^{42}$. We anticipate that the increased adoption of MRI technology in prostate cancer diagnostics and surveillance will enable us to monitor disease in a more reliable manner previously possible ${ }^{43}$. Then we will truly be able to determine the upgrading capacity of low-grade prostate cancer.

\section{Clinical outcomes of Gleason $3+3=6$ disease}

\section{Prostate-cancer-specific mortality}

Over the years, a considerable amount of data has accumulated regarding the risk associated with a Gleason pattern 3 attribution. Some important points need to be taken into account when evaluating these data. When we describe data on prostate cancer mortality it will, by necessity, be based on patients that were treated around 15-20 years ago, which has two implications. First, contemporary review of the original pathology results of these men would result 
in upgrading to a higher Gleason pattern in a moderate proportion of men. If the original assessment result was exclusive pattern 3 then the most probable upgrade would be to a Gleason score of $3+411$. Second, the precision of a biopsy two decades ago would have been substantially lower than today. We can, therefore, be certain that a considerable minority of men who were classified as having exclusive Gleason pattern 3 disease would have harboured a range of pattern 4 and an occasional pattern 5 tissues ${ }^{44}$. The implication is that the attribution of Gleason pattern 3 at that time was associated with considerably more risk than a contemporary attribution. This knowledge is useful to us in the epidemiological analysis of historical data sets. This systematic bias has the effect of rendering our estimations of risk on historical patients as the likely worst-case scenario as many of these men would have harboured Gleason score $>6$ if present-day grading systems were utilised. Increasing amounts of data demonstrate that patients with pure Gleason pattern 3 disease have negligible prostate-cancer-specific mortality. This assertion is very likely to be true and substantiated by the abundance of data of well characterized Gleason pattern 3 disease, which arises from the tens of thousands of men who have been monitored following a radical prostatectomy.

Peter Albertson was one of the first urologists to alert us to the very low propensity of a prostate-cancer-related death conferred by a historical attribution of Gleason pattern 3 status ${ }^{45}$. His report included data from men whose disease was given a Gleason score 3+3=6 between 1971 and 1984 and underwent conservative management. At 20 years, the prostate cancer death rate in this group was 30 deaths per 1000 person years - unchanged from the rates at 15 years. Investigators of another study reported on 223 untreated men with localized prostate cancer who were all initially managed with observation and androgen deprivation in the presence of symptomatic cancer progression. ${ }^{46}$ During a 30-year surveillance period, $12.3 \%$ of 65 patients diagnosed with Gleason score $\leq 6$ disease using the ISUP 2005 criteria died owing to their disease. This proportion translates into 1.4 men out of 1,000 men dying of Gleason score $\leq 6$ prostate cancer if monitored for a 10 -year period. 
A contemporary cohort of men with Gleason score 3+3=6 disease who opted for active surveillance had a cancer-specific survival of $>97 \%$ with median follow-up period of 22-82 months ${ }^{8}$. Furthermore, data that suggest inferior outcomes of these men who delay radical therapy is yet to be seen ${ }^{8}$. If the mortality in this cohort of men remains stable then, again, the question arises whether Gleason score $3+3=6$ prostate cancer results in any consequential cancer-specificmortality.

In a study from 2015, a team of investigators analyzed 1,829 prostatectomy specimens from patients treated between 1992 and 2008, acknowledging in their report that the pathological analyses were not performed according to 2005 ISUP criteria.47 In this cohort, 451 patients were identified as having Gleason score $3+3=6$ disease and their 10-year cancer-specific mortality was $0 \%$ and $0.5 \%$ in patients with $<0.5 \mathrm{ml}$ and $0.5-2.49 \mathrm{ml}$ disease volume, respectively. This difference in death rates were not statistically significant.

Abundant data exist of disease outcomes of tens of thousands of men with well characterized Gleason pattern 3 prostate cancer who have been monitored after radical prostatectomy ${ }^{48,49}$. A report on a cohort of nearly 10,000 men who were monitored following radical prostatectomy showed that the cancer-specific mortality was $0.03 \%$ in men who had pure Gleason pattern 3 disease in their radical specimens ${ }^{48}$.

The Prostate Cancer Intervention Versus Observation Trial (PIVOT) clearly demonstrated that men diagnosed with Gleason score $\leq 6$ prostate cancer do not reduce their risk of death (all-cause and cancer-specific mortality) at any interval if undergoing radical prostatectomy in comparison with observation 50 . Furthermore, men who were randomly assigned to surgery had a 15\% increased mortality compared with those assigned to observation for low-grade disease (determined by PSA level $\leq 10 \mathrm{ng} / \mathrm{ml}$, Gleason score $3+3=6$ and stage T1a-c or T2a).

Taking into account the imprecision of risk attribution in several of these cohorts and noting that, today, many of the investigated specimens would be classified as Gleason score $3+4=7$, this evidence collectively suggests that during a 1-20-year 
time period pure Gleason pattern 3 disease is associated with a minimal risk of a prostate-cancer-related death.

\section{Metastatic capability}

Most deaths from cancer occur as a result of metastasis and metastatic lymphatic involvement is a hallmark of advanced prostate cancer ${ }^{51,52}$. Presence of lymph node metastasis in patients with prostate cancer is directly associated with a poor prognosis and significantly decreased disease-specific and biochemicalrecurrence-free survival ${ }^{53}$. The notion that Gleason score $3+3=6$ disease has a substantial, or indeed any, ability to metastasise is increasingly being questioned 49,54 .

A large-scale population study published in 2014 investigated the presence of lymph node metastases in patients who underwent radical prostatectomy with lymph node sampling following a diagnosis of Gleason score 6 prostate cancer between 2004 and $2010^{54}$. Only $0.48 \%$ of 21,960 patients were found to have lymph node metastases. This proportion was reduced in data from large academic centres and the authors suggest that the presence of positive lymph nodes in patients diagnosed with Gleason score 6 disease could serve as a proxy for assessing the adherence to the 2005 ISUP criteria ${ }^{54}$.

Similarly, investigators in a study from 2012 used the 2005 ISUP 2005 Gleason scoring system to evaluate the potential of Gleason score $\leq 6$ disease to metastasize to lymph nodes ${ }^{49}$. Using data from 14,123 patients who had undergone radical prostatectomy at four large academic centres, the authors identified 22 patients with a positive lymph node. Histopathological re-review was undertaken in 19 of these patients demonstrating that all had a primary, secondary or tertiary Gleason pattern 4 or pattern 5; specimens from three patients were unavailable for re-review and the study was limited by a potential observer bias, as all involved pathologists were aware of the nodal metastatic status of the patients before re-review. In this setting, Gleason pattern 3 disease does not seem associated with a metastatic phenotype.

In patients with confirmed haematogenous prostate cancer metastasis, the skeletal system was affected in $90 \%$ of men ${ }^{55}$. However, even before ISUP 2005, 
$<1 \%$ of men diagnosed with Gleason score 3+3=6 disease were shown to have bone metastases ${ }^{56}$.

Another study in 178 men with Gleason score $3+3=6$ disease of $\leq 2.5 \mathrm{~cm}^{3}$ volume found a biochemical reccurrence rate of $0 \%$ following radical prostatectomy (median follow-up period 84 months) ${ }^{57}$.

These studies highlight that if a malignancy does not possess the ability to metastasize it fails to adhere to the label of lethal entity and, therefore, does not fulfil this classical attribute of cancer.

Two studies from 2013 have suggested that Gleason score 3+3=6 prostate cancer can indeed metastasize, resulting in lethal disease ${ }^{58,59}$. Nakabayashi et al. ${ }^{58}$ highlighted the small number of Gleason score $3+3=6$ prostate cancer that develops into lethal disease. In their cohort of 662 men, seven men were classified as having died from disease of this grade. Numerous methodological flaws of this study have been pointed out, including a lack of centralized review and absence of the exact characterization of the biopsy samples and pathological techniques used 60 . All these highlighted weaknesses have been accepted by the authors as a limitation to the validity of their conclusion 61.

The widely referenced study by Haffner et al. ${ }^{59}$ described a lethal metastatic clone originating from a low-grade primary tumour lesion. Using whole-genome sequencing and molecular pathological analyses the investigators demonstrated that the metastases arose from a focus of Gleason score $3+3=6$ disease. This particular patient did also harbour Gleason pattern 4 disease, but comprehensive sequencing of multiple tumour foci could not be performed, as the prostatic DNA was 20 years old. Several groups have raised concerns regarding the validity of the authors' findings based on data from a single patient ${ }^{62,63}$.

\section{Molecular changes of Gleason patterns}

In their seminal papers published in 2000 and 2011, Hanahan and Weinberg described the pathogenesis of cancer in six, and subsequently eight, pertinent hallmarks applicable to all variations of this disease ${ }^{64,65}$. We now have mounting molecular, proteomic and RNA-based evidence demonstrating that Gleason score $3+3=6$ prostate cancer is either failing to adhere to or to possess each of the six 
original hallmarks of cancer (TAB 1$)^{66}$. For higher-grade, however, an abundance of evidence exists that those diseases fulfil all six criteria66.

Research into the molecular bases of cancers has identified differing and exclusive pathologies between the various grades of numerous genitourinary malignancies ${ }^{67-69}$. For prostate cancer, genetic and epigenetic evidence is emerging that Gleason score $3+3=6$ disease is a distinct entity in comparison with its higher-grade counterparts $66,70-72$.

The evolution of normal prostatic tissue to high-grade prostate cancer involves morphological, chromosomal and developmental alterations. Germline mutations and environmental factors lead to synergistic DNA damage and oncogenic mutations which, in turn, prime prostatic tissue to transform into a malignant state. The combination of mutations of intracellular tumour suppressor genes (for example, PTEN and TP53), overexpression of oncogenes (for example, MYC, AKt/mTOR and genes encoding other tyrosine kinases) and oncogenic androgen-sensitive fusion products of chromosomal rearrangements (for example, TMPRSS2 and ERG) leads to uncontrolled cell proliferation ${ }^{73}$.

Genomic analysis of mRNA expression levels of pure Gleason pattern 3 in comparison with Gleason pattern 4 disease has demonstrated that Gleason pattern 3 tissues are relatively homogenous in the genes that are overexpressed $^{74,75}$. By contrast, mRNA expression patterns of Gleason pattern 4 tissue are unequivocally varied and incorporate a wide range of overexpressed genes $^{74,75}$. For Gleason pattern 3 disease, little evidence exists for the presence of subclonal driver mutations and, instead, these tissues have been shown to overexpress genes that primarily control intracellular metabolism. Mutations in Gleason pattern 4 disease is broadly linked to the overexpression of genes associated with the molecular traits of cancer; that is, those regulating the cell cycle, DNA replication and androgen signalling attenuation ${ }^{76}$.

Studying multifocal cancers, one group of researchers demonstrated that Gleason score $3+3=6$ prostate cancer diverges early from its higher-grade and metastatic counterparts ${ }^{70}$. Using laser microdissection and exome sequencing, they found that only $9 \%$ of high-confidence somatic mutations in Gleason score $3+3=6$ disease were shared with higher-grade disease, whereas the proportion 
was $82 \%$ when comparing high-grade and metastatic foci from lymph nodes. Notably, mutations in cancer-associated genes and in the tumour suppressor p53 signalling pathway were found exclusively in high-grade foci and metastases.

On a molecular level, using genome-wide DNA sequencing, morphologically normal tissue distant from the primary prostate cancer lesion has been shown to contain high levels of mutations ${ }^{77}$. This finding suggests that the underlying mutational processes in morphologically normal and low-grade prostate cancer tissue are also occurring in higher-grade disease consistent with a field effect. Protocols are in place to evaluate the mutation status in $>500$ men receiving standard-of-care treatment for castration-resistant disease which is hoped to provide a systematic approach to define the broader spectrum of genomic alterations in progressive lethal disease ${ }^{78}$. These studies might provide a systematic basis to define the broad spectrum of genomic alterations in progressive, lethal disease - an essential step in translating early laboratorybased observations into benefits in patient care ${ }^{78}$. Molecular characterization of prostate cancer is not expected to replace current clinical and pathological information. Instead, incorporation of these additional molecular data into current models might provide a more bespoke treatment plan for each patient ${ }^{78,67}$.

\section{Parallels with other cancers}

Keeping in mind previous adaptations in other genitourinary cancer, the loss of the cancer terminology for pure Gleason pattern 3 prostate cancer certainly would not be a radical step. Furthermore, this change would be in keeping with modifications made for several other solid-organ cancers. For example, numerous calls for ductal carcinoma in situ of the breast to forgo the label of carcinoma have been made, primarily led by a multidisciplinary NIH consensus ${ }^{79,80}$. Similar to Gleason pattern 3 prostate cancer this call for relabeling is fundamentally driven by the favourable prognosis and behaviour of this particular type of breast cancer.

In the field of endocrine surgery, a subset of papillary carcinoma of the thyroid has been labelled papillary microcarcinoma of the thyroid (PMCT), owing to its relatively indolent behaviour. Similar to Gleason score 3+3=6 prostate cancer, 
diagnoses of PMCT have increased, mainly because of more frequent use of ultrasound-guided fine-needle aspiration biopsy. Numerous studies and national guidelines have advocated discharging patients diagnosed with PMCT from future follow-up monitoring or, at most, mere observation as an appropriate management plan as opposed to invasive surgical therapy ${ }^{81-83}$.

Over the past several decades, an increasing number of people with chronic nonmalignant conditions, such as diabetes, hypertension and hypercholesterolaemia, are considered to be diseased, owing to lowered threshold values for diagnosis ${ }^{84}$. Now, evidence is emerging that instigating treatment modalities for the new diagnoses of these diseases is failing to reduce morbidity and mortality ${ }^{85-87}$.

Urologists specializing in the treatment of superficial bladder cancer succeeded in reclassifying a cancer phenotype to a noncancer phenotype. Papillary urothelial neoplasia of low malignant potential (PUNLMP) was previously categorized within the spectrum of grade 1 bladder cancer. The 1998 WHOISUP meeting recognized and renamed certain low-grade bladder tumours with a particularly good prognosis as PUNLMPs, avoiding the label of cancer ${ }^{88}$. PUNLMPs have been shown to have long-term nonmalignant capacity, as they lack any ability to become muscle-invasive or lethal disease according to the literature, thus, they continue to hold the status of a noncancer entity ${ }^{89}$.

Clinical comparisons can be made between PUNLMPs in the bladder and Gleason pattern 3 lesions in the prostate, but on a cellular level differences between the two tumours exist. Gleason pattern 3 disease demonstrates nuclear atypia of a malignant type and no basal cell layer that can be observed for its counterparts with higher grades, which in turn pathologically labels Gleason pattern $>3$ disease as an invasive disease. Histologically, PUNLMPs resemble an exophytic urothelial papilloma with an increased cellular proliferation that exceeds the thickness of normal urothelium ${ }^{90}$. Their papillary structures are lined by an organized arrangement of urothelial cells that have minimal architectural abnormalities and minimal nuclear atypia ${ }^{90}$.

\section{Overtreatment, costs and fear}


Gleason score 3+3=6 prostate cancer has been given numerous labels, including low-risk disease, insignificant disease, minimal-risk lesions and indolent lesions

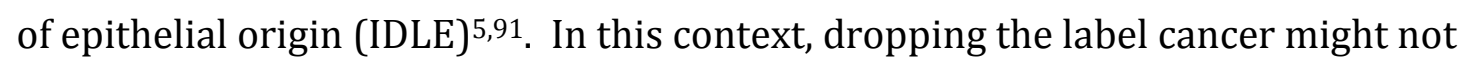
be a radical step.

In oncology, overdiagnosis is defined as the diagnosis of cancers that will not clinically manifest itself during an individual's lifetime or kill their carrier ${ }^{92}$. Overdiagnosis naturally leads to overtreatment. Little debate exists among experts in the field that the current generation of urologists are overtreating prostate cancer ${ }^{93-96}$. Clinicians are aware that the lifetime risk of substantial morbidity and mortality of a patient who has been diagnosed with Gleason score $3+3=6$ prostate cancer is minimal. However, when given this diagnosis most men still choose to undergo definitive treatment, despite the evidence that they will hardly benefit ${ }^{94,95,97}$. Now, there are signs that this behaviour is starting to change and that strategies such as active surveillance are being more widely adopted than was previously ${ }^{98}$. The reasons for this development are complex and include deeply entrenched societal and cultural drivers, as well as more widely acknowledged financial and medico-legal imperatives to treat $97,99,100$.

Overtreatment occurs in $67 \%$ of men diagnosed with low-risk prostate cancer and is positively associated with being married and residing in affluent areas ${ }^{97}$. One study found that $64.3 \%$ of a cohort of 39,803 men diagnosed with Gleason score $\leq 6$ disease underwent some form of aggressive treatment ${ }^{101}$. Receiving aggressive treatment was significantly associated with men who had a high socioeconomic status, were Caucasian or married. The findings of this study have led to calls for future policies to provide more rigid guidelines for the deferred treatment of Gleason score 3+3=6 prostate cancer and to offer more culturally and sociodemographically tailored counselling for those men at greatest risk of overtreatment ${ }^{101}$. Calculations for the USA show that the ability to avoid treating men with low-grade prostate cancer who will never succumb to their disease will save US\$1.32 billion alone and, more importantly, avoid the marked detrimental effect on patients' quality of life owing to the treatment modalities $^{97}$. 
A fear of litigation associated with relabeling Gleason score $3+3=6$ prostate cancer as noncancer may occur with pathologists and urologists ${ }^{100}$. Indeed, the most common reason for nonoperative claims in urology is related to the failure to diagnose or treat cancer ${ }^{102}$. Concerns have been raised that pathologists could potentially be a new group of clinicians who are regularly affected by medical liability claims ${ }^{100,103 .}$

The majority of men visiting their urologist because of lower urinary tract symptoms are worried about a diagnosis of prostate cancer above all else ${ }^{104}$. When a man is diagnosed with Gleason score $3+3=6$ disease psychological factors of the patient have been shown to drive disease management towards radical treatment ${ }^{105}$. Men of any age have an inherent fear of dying when faced with a diagnosis of prostate cancer ${ }^{100,106}$. The question arises whether a substantial number of men would still choose to undergo radical treatment if they were presented with the same prognostic and trifecta statistics but dropping the word cancer from their diagnosis of Gleason 3+3=6 disease.

\section{The grade group system}

The most recent modification to the Gleason score grading system was an attempt to create a five-point scale to reflect the range of risk ${ }^{107}$. We welcome this new system and look forward to its implementation. The new system comprises a five-stage ordinal scale: exclusive Gleason pattern 3 is categorized as prognostic Gleason grade group 1; group 2 comprises disease in which a secondary pattern 4 is present in a predominant pattern 3; group 3 includes tumours in which pattern 4 dominates over pattern 3; group 4 is attributed to Gleason score 8 disease; and group 5 is reserved for Gleason score $\geq 9$ disease.

\section{Conclusions}

We propose that our colleagues at ISUP further modify the classification of prostate cancer and drop the label cancer for Gleason score 3+3=6 disease. With the increased adoption of image-guided techniques to obtain prostatic tissue in a more accurate manner, the fear of potentially having missed disease of an increased grade will not bias disease reporting. 
When a patient is given a diagnosis of well characterized Gleason score $3+3=6$ prostate cancer, he has been labelled with a disease status that is associated with a higher mortality if treated compared with leaving it untreated. In addition, the consequences of treatment are considerable both in economic terms and in its inevitable harms; few men get through their treatments without sustaining some harm. Furthermore, the disease entity that we are describing is distinct from its more aggressive counterpart both morphologically and by modern molecular characterisation. Yet, we insist on using the same descriptor for a disease with a broad range of risk and also invoke the same word that is linked with 50\% 1year survival seen in some cancers, such as pancreatic and lung cancer. The move to a five-stage ordinal scale that places exclusive Gleason pattern 3 disease on the lowest rung of the ladder of risk is a welcome and timely development.

Some of our colleagues who have opposing views might be apprehensive owing to biopsy sampling imprecision and reproducibility, especially when giving a certain diagnosis of a 'benign' condition following assessment of a needle biopsy sample. Furthermore, in pathological terms, Gleason pattern 3 fulfils multiple criteria of cancer. The large and varied evidence that has accumulated over the past decades combined with the reduction in overall risk associated with Gleason pattern 3 (owing to reclassification) and modern diagnostic precision (virtually eliminating upgrading at radical prostatectomy) suggests that the time has come to relegate well characterized Gleason pattern 3 morphological change as a potential risk factor for cancer - namely Gleason pattern 4 and pattern 5 and not cancer in its own right.

\section{Acknowledgements}

Mark Emberton holds Senior Investigator status with the UK National Institute for Health Research (NIHR). He receives research support from the NIHR Biomedical Research Centre at University College London Hospitals NHS Foundation Trust and University College London. 


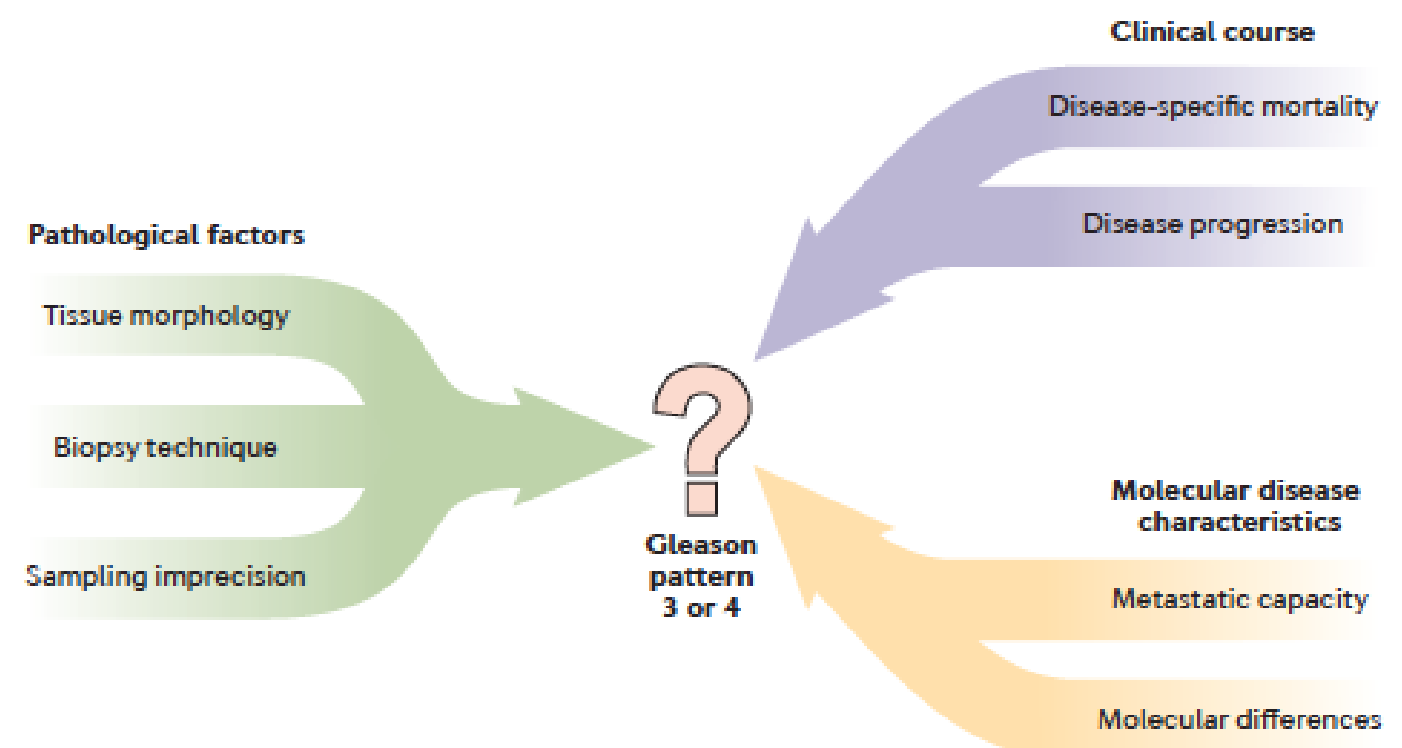

Figure 1. The pathological, clinical and molecular differences between Gleason pattern 3 and 4 . The distinction between Gleason pattern 3 and pattern 4 is evident in their heterogeneous molecular characteristics and distinct clinical courses. Factors of pathological assessment must also be taken into consideration when differentiating these two patterns of disease. 


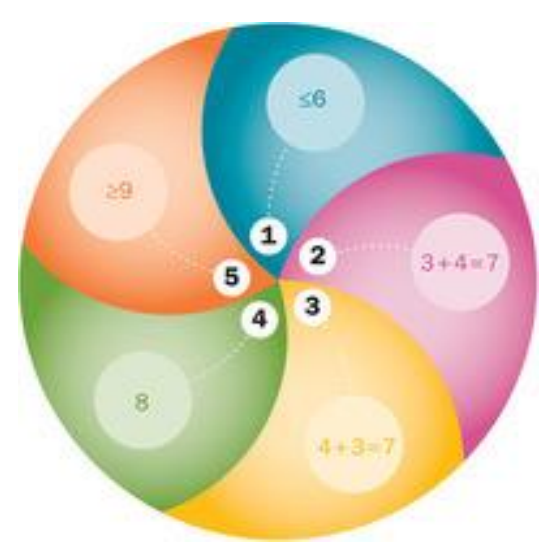

Figure 2. The five-point grade group system of Gleason scoring. The most recent modification to the Gleason grading system resulted in a five-point scale reflecting disease risk groups. Grade group 1 are tissues with exclusive Gleason pattern3; group 2 are diseases with predominant pattern 3 but secondary pattern 4; group 3 includes those in which pattern 4 dominates over pattern 3; group 4 is attributed to Gleason score 8 disease; group 5 is reserved for Gleason score $\geq 9$ disease. 
TAB 1 | Molecular distinction of Gleason pattern 3 or score 6 tissue from highergrade disease and correlation to the six hallmarks of cancer.

\begin{tabular}{|c|c|c|}
\hline Hallmark & Study & Findings \\
\hline \multirow[t]{2}{*}{$\begin{array}{l}\text { 1. Self-sufficiency in } \\
\text { growth signals }\end{array}$} & Ross et al.108 & $\begin{array}{l}\text { Exclusive differential expression of } 670 \text { genes between } \\
\text { Gleason pattern } 3 \text { and pattern } 4 \text { tissues, including } E G F \text {, } \\
E G F R, M A P 2 K 4, R A L A, R E P S 2, P H L P P, P M L \text {, which are all } \\
\text { established genes associated with growth signalling }\end{array}$ \\
\hline & Skacel et al. ${ }^{109}$ & $\begin{array}{l}\text { Amplification of } E R B B 2 \text {, - a known proto-oncogene, in } \\
\text { Gleason pattern } 4 \text { compared with pattern } 3 \text { tissue }\end{array}$ \\
\hline $\begin{array}{l}\text { 2. Insensitivity to } \\
\text { antigrowth signals }\end{array}$ & Padar et al. ${ }^{110}$ & $\begin{array}{l}\text { Significantly higher frequency of } C C N D 2 \text {, involved in } \\
\text { regulation of cell-cycle G1 to } \mathrm{S} \text { phase transition, } \\
\text { methylation in Gleason pattern } 3 \text { compared with higher- } \\
\text { grade tissue }\end{array}$ \\
\hline \multirow[t]{2}{*}{$\begin{array}{l}\text { 3. Evading } \\
\text { apoptosis }\end{array}$} & $\begin{array}{l}\text { Fleischmann } \\
\text { et al. }{ }^{111}\end{array}$ & $\begin{array}{l}\text { Significant upregulation of antiapoptotic } B C L 2 \text { in higher- } \\
\text { grade compared with Gleason pattern } 3 \text { tissue }\end{array}$ \\
\hline & True et al. ${ }^{75}$ & $\begin{array}{l}\text { Greater expression of antiapoptotic DAD- } 1 \text { in higher- } \\
\text { grade compared with Gleason score } 6 \text { tissue }\end{array}$ \\
\hline \multirow[t]{2}{*}{$\begin{array}{l}\text { 4. Unlimited } \\
\text { replicative } \\
\text { potential }\end{array}$} & Tomlins et al. ${ }^{76}$ & $\begin{array}{l}\text { Compared with Gleason pattern } 3 \text { tissue, pattern } 4 \text { tissue } \\
\text { showed an attenuated androgen signalling signature, } \\
\text { similar to metastatic prostate cancer }\end{array}$ \\
\hline & $\begin{array}{l}\text { Hendriksen et } \\
\quad \text { al. }{ }^{112}\end{array}$ & $\begin{array}{l}\text { Of } 200 \text { androgen-responsive genes in prostate cancer } \\
\text { functionally distinct parts of the androgen receptor } \\
\text { pathway were downregulated in high-grade cancers, } \\
\text { including decreased HERPUD1, STK39, DHCR } 24 \text { and } \\
\text { SOCS } 2 \text { mRNA expression }\end{array}$ \\
\hline $\begin{array}{l}\text { 5. Sustained } \\
\text { angiogenesis }\end{array}$ & West et al.113 & $\begin{array}{l}\text { Upregulation of proangiogenic vascular endothelial } \\
\text { growth factor in higher-grade compared with Gleason } \\
\text { pattern } 3 \text { tissue }\end{array}$ \\
\hline $\begin{array}{l}\text { 6. Tissue invasion } \\
\text { and metastasis }\end{array}$ & $\begin{array}{l}\text { de Muga et } \\
\text { al.114 }\end{array}$ & $\begin{array}{l}\text { Upregulation of } C X C R 4 \text {, involved in directing cancer cell } \\
\text { migration to metastatic sites, in Gleason score }>7 \text { tissue } \\
\text { independent of TMPRSS2-ERG rearrangement }\end{array}$ \\
\hline
\end{tabular}


Saiful Miah gained his MBChB from the University of Glasgow, UK, and is currently a NIHR clinical lecturer in urology at the Division of Surgery and Interventional Science, University College London, UK. He holds a PhD from the University of Sheffield for his thesis titled "The role of microRNA in bladder cancer".

Hashim U. Ahmed gained his MBChB from the University of Oxford, St Peter's College, UK. He currently holds an Medical Research Council clinician-scientist fellowship and is a reader in urology at University College London, UK. His research interests are in health technology evaluation, including multiparametric MRI, histoscanning and tissue type imaging, as well as therapeutic interventions, such as high-intensity focused ultrasound, irreversible electroporation, photodynamic therapy, cryotherapy and radiofrequency ablation. Mr Ahmed was involved in numerous phase I/II clinical trials evaluating the role of focal therapy in prostate cancer treatment and is lead principal investigator and co-principal investigator of a number of multicentre trials.

Alex Freeman gained his MBBS from St George's Medical School, London, UK. He is a Consultant Histopathologist with University College London Hospitals (UCLH) NHS Foundation Trust. He trained mainly at UCLH, with attachments to Royal Brompton Hospital and Royal Marsden Hospital. He spent two years at Addenbrooke's Hospital, Cambridge, UK, completing an MD thesis with Professor Ron Laskey in molecular biology. His main areas of interest are urological pathology and dermatopathology with over 200 peer-reviewed publications to his name.

Professor Mark Emberton gained his MBBS from Imperial College at St Mary's Campus, London, UK. He is the Professor of Interventional Oncology, Division of Surgery and Interventional Science, University College London, Clinical Director of the Clinical Effectiveness Unit, Royal College of Surgeons of England. He has been recently appointed the Dean of the Faculty of University College London Medical Sciences. His main interests lie in improving diagnostic and therapeutic pathways for men with prostate cancer, principally through the use of novel imaging techniques and minimally invasive treatments. 


\section{References}

1 Jemal, A. et al. Cancer statistics, 2006. CA Cancer J Clin 56, 106-130 (2006).

2 Humphrey, P. A. Gleason grading and prognostic factors in carcinoma of the prostate. Mod Pathol 17, 292-306, doi:10.1038/modpathol.3800054 (2004).

3 Gleason, D. F. Classification of prostatic carcinomas. Cancer Chemother Rep 50, 125-128 (1966).

$4 \mathrm{Xu}, \mathrm{W} . \&$ Zhou, M. A concise update on prostate pathology. Cesk Patol 50, 120-128 (2014).

5 Esserman, L., Shieh, Y. \& Thompson, I. Rethinking screening for breast cancer and prostate cancer. Jama 302, 1685-1692, doi:10.1001/jama.2009.1498 (2009).

6 Nickel, J. C. \& Speakman, M. Should we really consider Gleason 6 prostate cancer? BJU Int 109, 645-646, doi:10.1111/j.1464-410X.2011.10854.x (2012).

$7 \quad$ Klotz, L. Cancer overdiagnosis and overtreatment. Curr Opin Urol 22, 203209, doi:10.1097/MOU.0b013e32835259aa (2012).

8 Lepor, H. \& Donin, N. M. Gleason 6 prostate cancer: serious malignancy or toothless lion? Oncology (Williston Park) 28, 16-22 (2014).

9 Gleason, D. F. \& Mellinger, G. T. Prediction of prognosis for prostatic adenocarcinoma by combined histological grading and clinical staging. $J$ Urol 111, 58-64 (1974).

10 Delahunt, B., Miller, R. J., Srigley, J. R., Evans, A. J. \& Samaratunga, H. Gleason grading: past, present and future. Histopathology 60, 75-86, doi:10.1111/j.1365-2559.2011.04003.x (2012).

11 Montironi, R. et al. Original Gleason system versus 2005 ISUP modified Gleason system: the importance of indicating which system is used in the patient's pathology and clinical reports. Eur Urol 58, 369-373, doi:10.1016/j.eururo.2010.04.028 (2010).

12 Allsbrook, W. C., Jr. et al. Interobserver reproducibility of Gleason grading of prostatic carcinoma: urologic pathologists. Hum Pathol 32, 74-80, doi:10.1053/hupa.2001.21134 (2001).

13 Oyama, T. et al. A comparison of interobserver reproducibility of Gleason grading of prostatic carcinoma in Japan and the United States. Arch Pathol Lab Med 129, 1004-1010, doi:10.1043/15432165(2005)129[1004:ACOIRO]2.0.CO;2 (2005).

14 Shah, R. B. Current perspectives on the Gleason grading of prostate cancer. Arch Pathol Lab Med 133, 1810-1816, doi:10.1043/1543-2165133.11.1810 (2009).

15 Epstein, J. I. An update of the Gleason grading system. J Urol 183, 433-440, doi:10.1016/j.juro.2009.10.046 (2010).

16 Epstein, J. I. Gleason score 2-4 adenocarcinoma of the prostate on needle biopsy: a diagnosis that should not be made. Am J Surg Pathol 24, 477-478 (2000). 
17 Ghani, K. R., Grigor, K., Tulloch, D. N., Bollina, P. R. \& McNeill, S. A. Trends in reporting Gleason score 1991 to 2001: changes in the pathologist's practice. Eur Urol 47, 196-201, doi:10.1016/j.eururo.2004.07.029 (2005).

18 Helpap, B. \& Egevad, L. The significance of modified Gleason grading of prostatic carcinoma in biopsy and radical prostatectomy specimens. Virchows Arch 449, 622-627, doi:10.1007/s00428-006-0310-6 (2006).

19 Epstein, J. I., Allsbrook, W. C., Jr., Amin, M. B. \& Egevad, L. L. The 2005 International Society of Urological Pathology (ISUP) Consensus Conference on Gleason Grading of Prostatic Carcinoma. Am J Surg Pathol 29, 1228-1242 (2005).

20 Albertsen, P. C. et al. Prostate cancer and the Will Rogers phenomenon. $J$ Natl Cancer Inst 97, 1248-1253, doi:10.1093/jnci/dji248 (2005).

21 Berg, K. D. et al. The impact of the 2005 International Society of Urological Pathology consensus guidelines on Gleason grading - a matched-pair analysis. BJU Int 117, 883-889, doi:10.1111/bju.13439 (2016).

22 Silletti, J. P., Gordon, G. J., Bueno, R., Jaklitsch, M. \& Loughlin, K. R. Prostate biopsy: past, present, and future. Urology 69, 413-416, doi:10.1016/j.urology.2007.01.096 (2007).

23 Eichler, K. et al. Diagnostic value of systematic biopsy methods in the investigation of prostate cancer: a systematic review. J Urol 175, 16051612, doi:10.1016/S0022-5347(05)00957-2 (2006).

24 Mai, Z. et al. Transperineal template-guided prostate biopsy: 10 years of experience. BJU Int, doi:10.1111/bju.13024 (2014).

25 Moore, C. M. et al. Image-guided prostate biopsy using magnetic resonance imaging-derived targets: a systematic review. Eur Urol 63, 125140, doi:10.1016/j.eururo.2012.06.004 (2013).

26 Shariat, S. F. \& Roehrborn, C. G. Using biopsy to detect prostate cancer. Rev Urol 10, 262-280 (2008).

27 Bostwick, D. G. Evaluating prostate needle biopsy: therapeutic and prognostic importance. CA Cancer J Clin 47, 297-319 (1997).

28 Shaw, G. L. et al. Identification of pathologically insignificant prostate cancer is not accurate in unscreened men. Br J Cancer 110, 2405-2411, doi:10.1038/bjc.2014.192 (2014).

29 Kasivisvanathan, V. et al. Transperineal magnetic resonance image targeted prostate biopsy versus transperineal template prostate biopsy in the detection of clinically significant prostate cancer. J Urol 189, 860-866, doi:10.1016/j.juro.2012.10.009 (2013).

30 Siddiqui, M. M. et al. Magnetic resonance imaging/ultrasound-fusion biopsy significantly upgrades prostate cancer versus systematic 12-core transrectal ultrasound biopsy. Eur Urol 64, 713-719, doi:10.1016/j.eururo.2013.05.059 (2013).

31 Fine, S. W. \& Epstein, J. I. A contemporary study correlating prostate needle biopsy and radical prostatectomy Gleason score. J Urol 179, 13351338; discussion 1338-1339, doi:10.1016/j.juro.2007.11.057 (2008).

32 Capitanio, U. et al. Biopsy core number represents one of foremost predictors of clinically significant gleason sum upgrading in patients with low-risk prostate cancer. Urology 73, 1087-1091, doi:10.1016/j.urology.2008.10.048 (2009). 
33 Epstein, J. I., Feng, Z., Trock, B. J. \& Pierorazio, P. M. Upgrading and downgrading of prostate cancer from biopsy to radical prostatectomy: incidence and predictive factors using the modified Gleason grading system and factoring in tertiary grades. Eur Urol 61, 1019-1024, doi:10.1016/j.eururo.2012.01.050 (2012).

34 Epstein, J. I., Sanderson, H., Carter, H. B. \& Scharfstein, D. O. Utility of saturation biopsy to predict insignificant cancer at radical prostatectomy. Urology 66, 356-360, doi:10.1016/j.urology.2005.03.002 (2005).

35 Sved, P. D., Gomez, P., Manoharan, M., Kim, S. S. \& Soloway, M. S. Limitations of biopsy Gleason grade: implications for counseling patients with biopsy Gleason score 6 prostate cancer. J Urol 172, 98-102, doi:10.1097/01.ju.0000132135.18093.d6 (2004).

36 Emiliozzi, P. et al. Increased accuracy of biopsy Gleason score obtained by extended needle biopsy. J Urol 172, 2224-2226 (2004).

37 Nayyar, R. et al. Upgrading of Gleason score on radical prostatectomy specimen compared to the pre-operative needle core biopsy: an Indian experience. Indian J Urol 26, 56-59, doi:10.4103/0970-1591.60445 (2010).

38 Marks, L., Young, S. \& Natarajan, S. MRI-ultrasound fusion for guidance of targeted prostate biopsy. Curr Opin Urol 23, 43-50, doi:10.1097/MOU.0b013e32835ad3ee (2013).

39 Ahmed, H. U. The PROMIS of MRI. BJU Int 118, 7, doi:10.1111/bju.13547 (2016).

40 Griffiths, D. F. et al. A study of Gleason score interpretation in different groups of UK pathologists; techniques for improving reproducibility. Histopathology 48, 655-662, doi:10.1111/j.1365-2559.2006.02394.x (2006).

41 Penney, K. L. et al. Gleason grade progression is uncommon. Cancer Res 73, 5163-5168, doi:10.1158/0008-5472.CAN-13-0427 (2013).

42 Sonn, G. A. et al. Initial experience with electronic tracking of specific tumor sites in men undergoing active surveillance of prostate cancer. Urol Oncol 32, 952-957, doi:10.1016/j.urolonc.2014.04.003 (2014).

43 Robertson, N. L. et al. MAPPED study design: a 6 month randomised controlled study to evaluate the effect of dutasteride on prostate cancer volume using magnetic resonance imaging. Contemp Clin Trials 34, 80-89, doi:10.1016/j.cct.2012.10.003 (2013).

44 Mufarrij, P., Sankin, A., Godoy, G. \& Lepor, H. Pathologic outcomes of candidates for active surveillance undergoing radical prostatectomy. Urology 76, 689-692, doi:10.1016/j.urology.2009.12.075 (2010).

45 Albertsen, P. C., Hanley, J. A. \& Fine, J. 20-year outcomes following conservative management of clinically localized prostate cancer. Jama 293, 2095-2101, doi:10.1001/jama.293.17.2095 (2005).

46 Popiolek, M. et al. Natural history of early, localized prostate cancer: a final report from three decades of follow-up. Eur Urol 63, 428-435, doi:10.1016/j.eururo.2012.10.002 (2013).

47 Schiffmann, J. et al. Tumor volume in insignificant prostate cancer: increasing threshold gains increasing risk. Prostate 75, 45-49, doi:10.1002/pros.22889 (2015). 
48 Eggener, S. E. et al. Predicting 15-year prostate cancer specific mortality after radical prostatectomy. $J$ Urol 185, 869-875, doi:10.1016/j.juro.2010.10.057 (2011).

49 Ross, H. M. et al. Do adenocarcinomas of the prostate with Gleason score (GS) </=6 have the potential to metastasize to lymph nodes? Am J Surg Pathol 36, 1346-1352, doi:10.1097/PAS.0b013e3182556dcd (2012).

50 Wilt, T. J. et al. Radical prostatectomy versus observation for localized prostate cancer. N Engl J Med 367, 203-213, doi:10.1056/NEJMoa1113162 (2012).

51 Brower, V. Researchers tackle metastasis, cancer's last frontier. J Natl Cancer Inst 99, 109-111, doi:10.1093/jnci/djk047 (2007).

52 Datta, K., Muders, M., Zhang, H. \& Tindall, D. J. Mechanism of lymph node metastasis in prostate cancer. Future Oncol 6, 823-836, doi:10.2217/fon.10.33 (2010).

53 Cai, T. et al. Clinical importance of lymph node density in predicting outcome of prostate cancer patients. J Surg Res 167, 267-272, doi:10.1016/j.jss.2009.05.004 (2011).

54 Liu, J. J. et al. Nationwide prevalence of lymph node metastases in Gleason score $3+3=6$ prostate cancer. Pathology 46, 306-310, doi:10.1097/PAT.0000000000000097 (2014).

55 Bubendorf, L. et al. Metastatic patterns of prostate cancer: an autopsy study of 1,589 patients. Hum Pathol 31, 578-583 (2000).

56 Lee, N. et al. Which patients with newly diagnosed prostate cancer need a radionuclide bone scan? An analysis based on 631 patients. Int J Radiat Oncol Biol Phys 48, 1443-1446 (2000).

57 Ting, F. et al. Tumor volume in insignificant prostate cancer: Increasing the threshold is a safe approach to reduce over-treatment. Prostate, doi:10.1002/pros.23062 (2015).

58 Nakabayashi, M. et al. Clinical predictors of survival in men with castration-resistant prostate cancer: evidence that Gleason score 6 cancer can evolve to lethal disease. Cancer 119, 2990-2998, doi:10.1002/cncr.28102 (2013).

59 Haffner, M. C. et al. Tracking the clonal origin of lethal prostate cancer. J Clin Invest 123, 4918-4922, doi:10.1172/JCI70354 (2013).

60 El-Shater Bosaily, A., Arya, M. \& Ahmed, H. Clinical predictors of survival in men with castration-resistant prostate cancer: evidence that Gleason score 6 cancer can evolve to lethal disease. Cancer 119, 4052-4053, doi:10.1002/cncr.28333 (2013).

61 Hayes, J. H., Nakabayashi, M. \& Kantoff, P. W. Reply to clinical predictors of survival in men with castration-resistant prostate cancer: evidence that Gleason score 6 cancer can evolve to lethal disease. Cancer 119, 4053, doi:10.1002/cncr.28332 (2013).

62 Passoni, N. M. \& Polascik, T. J. Words of wisdom. Re: Tracking the clonal origin of lethal prostate cancer. Eur Urol 66, 390-391, doi:10.1016/j.eururo.2014.05.022 (2014).

63 Brannon, A. R. \& Sawyers, C. L. "N of 1" case reports in the era of wholegenome sequencing. J Clin Invest 123, 4568-4570, doi:10.1172/JCI70935 (2013). 
64 Hanahan, D. \& Weinberg, R. A. The hallmarks of cancer. Cell 100, 57-70 (2000).

65 Hanahan, D. \& Weinberg, R. A. Hallmarks of cancer: the next generation. Cell 144, 646-674, doi:10.1016/j.cell.2011.02.013 (2011).

66 Ahmed, H. U., Arya, M., Freeman, A. \& Emberton, M. Do low-grade and low-volume prostate cancers bear the hallmarks of malignancy? Lancet Oncol 13, e509-517, doi:10.1016/S1470-2045(12)70388-1 (2012).

67 Miah, S. \& Catto, J. W. MicroRNA in prostate cancer: an opportunity to individualize patient care. The Journal of urology 187, 1155-1156, doi:10.1016/j.juro.2012.01.028 (2012).

68 Miah, S., Pang, K. \& Catto, J. W. MicroRNA and urothelial cell carcinoma. BJU Int 113, 811-812, doi:10.1111/bju.12689 (2014).

69 Catto, J. W. et al. Distinct microRNA alterations characterize high- and low-grade bladder cancer. Cancer Res 69, 8472-8481, doi:10.1158/00085472.CAN-09-0744 (2009).

70 VanderWeele, D. J. et al. Low-grade prostate cancer diverges early from high grade and metastatic disease. Cancer Sci 105, 1079-1085, doi:10.1111/cas.12460 (2014).

71 Lavery, H. J. \& Droller, M. J. Do Gleason patterns 3 and 4 prostate cancer represent separate disease states? J Urol 188, 1667-1675, doi:10.1016/j.juro.2012.07.055 (2012).

72 Berndt, S. I. et al. Two susceptibility loci identified for prostate cancer aggressiveness. Nat Commun 6, 6889, doi:10.1038/ncomms7889 (2015).

73 Shen, M. M. \& Abate-Shen, C. Molecular genetics of prostate cancer: new prospects for old challenges. Genes Dev 24, 1967-2000, doi:10.1101/gad.1965810 (2010).

74 Penney, K. L. et al. mRNA expression signature of Gleason grade predicts lethal prostate cancer. J Clin Oncol 29, 2391-2396, doi:10.1200/JC0.2010.32.6421 (2011).

75 True, L. et al. A molecular correlate to the Gleason grading system for prostate adenocarcinoma. Proc Natl Acad Sci U S A 103, 10991-10996, doi:10.1073/pnas.0603678103 (2006).

76 Tomlins, S. A. et al. Integrative molecular concept modeling of prostate cancer progression. Nat Genet 39, 41-51, doi:10.1038/ng1935 (2007).

77 Cooper, C. S. et al. Analysis of the genetic phylogeny of multifocal prostate cancer identifies multiple independent clonal expansions in neoplastic and morphologically normal prostate tissue. Nat Genet 47, 367-372, doi:10.1038/ng.3221 (2015).

78 Barbieri, C. E., Demichelis, F. \& Rubin, M. A. The lethal clone in prostate cancer: redefining the index. Eur Urol 66, 395-397, doi:10.1016/j.eururo.2013.12.052 (2014).

79 Allegra, C. J. et al. NIH state-of-the-science conference statement: diagnosis and management of ductal carcinoma in situ (DCIS). NIH Consens State Sci Statements 26, 1-27 (2009).

80 Allegra, C. J. et al. National Institutes of Health State-of-the-Science Conference statement: Diagnosis and Management of Ductal Carcinoma In Situ September 22-24, 2009. J Natl Cancer Inst 102, 161-169, doi:10.1093/jnci/djp485 (2010). 
81 Ito, Y. et al. An observation trial without surgical treatment in patients with papillary microcarcinoma of the thyroid. Thyroid 13, 381-387, doi:10.1089/105072503321669875 (2003).

82 Piersanti, M., Ezzat, S. \& Asa, S. L. Controversies in papillary microcarcinoma of the thyroid. Endocr Pathol 14, 183-191 (2003).

83 Perros, P. et al. Guidelines for the management of thyroid cancer. Clin Endocrinol (Oxf) 81 Suppl 1, 1-122, doi:10.1111/cen.12515 (2014).

84 Schwartz, L. M. \& Woloshin, S. Changing disease definitions: implications for disease prevalence. Analysis of the Third National Health and Nutrition Examination Survey, 1988-1994. Eff Clin Pract 2, 76-85 (1999).

85 Martin, S. A., Boucher, M., Wright, J. M. \& Saini, V. Mild hypertension in people at low risk. Bmj 349, g5432, doi:10.1136/bmj.g5432 (2014).

86 Schroeder, A. R. \& Redberg, R. F. Cholesterol screening and management in children and young adults should start early--NO! Clin Cardiol 35, 665668, doi:10.1002/clc.22075 (2012).

87 Lipska, K. J. et al. Potential overtreatment of diabetes mellitus in older adults with tight glycemic control. JAMA Intern Med 175, 356-362, doi:10.1001/jamainternmed.2014.7345 (2015).

88 Epstein, J. I., Amin, M. B., Reuter, V. R. \& Mostofi, F. K. The World Health Organization/International Society of Urological Pathology consensus classification of urothelial (transitional cell) neoplasms of the urinary bladder. Bladder Consensus Conference Committee. Am J Surg Pathol 22, 1435-1448 (1998).

89 Fujii, Y., Kawakami, S., Koga, F., Nemoto, T. \& Kihara, K. Long-term outcome of bladder papillary urothelial neoplasms of low malignant potential. BJU Int 92, 559-562 (2003).

90 MacLennan, G. T., Kirkali, Z. \& Cheng, L. Histologic grading of noninvasive papillary urothelial neoplasms. Eur Urol 51, 889-897; discussion 897-888, doi:10.1016/j.eururo.2006.10.037 (2007).

91 Bastian, P. J. et al. Insignificant prostate cancer and active surveillance: from definition to clinical implications. Eur Urol 55, 1321-1330, doi:10.1016/j.eururo.2009.02.028 (2009).

92 Moynihan, R., Doust, J. \& Henry, D. Preventing overdiagnosis: how to stop harming the healthy. Bmj 344, e3502, doi:10.1136/bmj.e3502 (2012).

93 Klotz, L. Prostate cancer overdiagnosis and overtreatment. Curr Opin Endocrinol Diabetes Obes 20, 204-209, doi:10.1097/MED.0b013e328360332a (2013).

94 Daskivich, T. J. et al. Variation in treatment associated with life expectancy in a population-based cohort of men with early-stage prostate cancer. Cancer 120, 3642-3650, doi:10.1002/cncr.28926 (2014).

95 Hoffman, K. E. et al. Physician variation in management of low-risk prostate cancer: a population-based cohort study. JAMA Intern Med 174, 1450-1459, doi:10.1001/jamainternmed.2014.3021 (2014).

96 Shao, Y. H. et al. Risk profiles and treatment patterns among men diagnosed as having prostate cancer and a prostate-specific antigen level below $4.0 \mathrm{ng} / \mathrm{ml}$. Arch Intern Med 170, 1256-1261, doi:10.1001/archinternmed.2010.221 (2010). 
97 Aizer, A. A. et al. Cost implications and complications of overtreatment of low-risk prostate cancer in the United States. J Natl Compr Canc Netw 13, 61-68 (2015).

98 van der Kwast, T. H. \& Roobol, M. J. Prostate cancer: Is prostatectomy for Gleason score 6 a treatment failure? Nat Rev Urol 12, 10-11, doi:10.1038/nrurol.2014.335 (2015).

99 Barry, J. M. Words of wisdom. Re: Radical prostatectomy versus observation for localized prostate cancer. Eur Urol 62, 1196, doi:10.1016/j.eururo.2012.09.016 (2012).

100 Carter, H. B. et al. Gleason score 6 adenocarcinoma: should it be labeled as cancer? J Clin Oncol 30, 4294-4296, doi:10.1200/JCO.2012.44.0586 (2012).

101 Mahal, B. A. et al. Who Bears the Greatest Burden of Aggressive Treatment of Indolent Prostate Cancer? Am J Med, doi:10.1016/j.amjmed.2014.12.030 (2015).

102 Osman, N. I. \& Collins, G. N. Urological litigation in the UK National Health Service (NHS): an analysis of 14 years of successful claims. BJU Int 108, 162-165, doi:10.1111/j.1464-410X.2011.10130.x (2011).

103 Dunn, I. B. \& Kirk, D. Legal pitfalls in the diagnosis of prostate cancer. BJU Int 86, 304-307 (2000).

104 Brown, C. T. et al. The fear of prostate cancer in men with lower urinary tract symptoms: should symptomatic men be screened? BJU Int 91, 30-32 (2003).

105 Berger, Z. D., Yeh, J. C., Carter, H. B. \& Pollack, C. E. Characteristics and experiences of patients with localized prostate cancer who left an active surveillance program. Patient 7, 427-436, doi:10.1007/s40271-0140066-z (2014).

106 Carrion, I. V., Nedjat-Haiem, F. R. \& Marquez, D. X. Examining cultural factors that influence treatment decisions: a pilot study of Latino men with cancer. J Cancer Educ 28, 729-737, doi:10.1007/s13187-013-0522-9 (2013).

107 Epstein, J. I. et al. A Contemporary Prostate Cancer Grading System: A Validated Alternative to the Gleason Score. Eur Urol, doi:10.1016/j.eururo.2015.06.046 (2015).

108 Ross, A. E. et al. Gene expression pathways of high grade localized prostate cancer. Prostate 71, 1568-1577, doi:10.1002/pros.21373 (2011).

109 Skacel, M. et al. Aneusomy of chromosomes 7, 8, and 17 and amplification of HER-2/neu and epidermal growth factor receptor in Gleason score 7 prostate carcinoma: a differential fluorescent in situ hybridization study of Gleason pattern 3 and 4 using tissue microarray. Hum Pathol 32, 13921397, doi:10.1053/hupa.2001.29676 (2001).

110 Padar, A. et al. Inactivation of cyclin D2 gene in prostate cancers by aberrant promoter methylation. Clin Cancer Res 9, 4730-4734 (2003).

111 Fleischmann, A. et al. Prognostic relevance of Bcl-2 overexpression in surgically treated prostate cancer is not caused by increased copy number or translocation of the gene. Prostate 72, 991-997, doi:10.1002/pros.21504 (2012). 
112 Hendriksen, P. J. et al. Evolution of the androgen receptor pathway during progression of prostate cancer. Cancer Res 66, 5012-5020, doi:10.1158/0008-5472.CAN-05-3082 (2006).

113 West, A. F., O'Donnell, M., Charlton, R. G., Neal, D. E. \& Leung, H. Y. Correlation of vascular endothelial growth factor expression with fibroblast growth factor-8 expression and clinico-pathologic parameters in human prostate cancer. $B r \quad J$ Cancer 85, 576-583, doi:10.1054/bjoc.2001.1971 (2001).

114 de Muga, S. et al. CXCR4 mRNA overexpression in high grade prostate tumors: lack of association with TMPRSS2-ERG rearrangement. Cancer Biomark 12, 21-30, doi:10.3233/CBM-2012-00288 (2012). 